\title{
Effect of metal groups in transition metal alkoxide catalysts on transesterification
}

\author{
Gayan Nawaratna, Sergio Capareda, Sandun D. Fernando* \\ Biological and Agricultural Engineering Department, 321 Scoates Hall, Texas A\&M University, College Station TX USA
}

\section{Email address:}

sfernando@tamu.edu (S. D. Fernando)

\section{To cite this article:}

Gayan Nawaratna, Sergio Capareda, Sandun D. Fernando. Effect of Metal Groups in Transition Metal Alkoxide Catalysts on Transesterification. Advances in Materials. Vol. 1, No. 1, 2012, pp. 1-8. doi: 10.11648/j.am.20120101.11

\begin{abstract}
Transesterification is a widely used chemical reaction in chemical industry. The use of common homogeneous catalysts that comprise of alkali metal hydroxides/alkoxides and inorganic acids lead to intricacies associated with post reaction separation of catalysts from products. On the other hand, heterogeneous catalysts possess the drawback of limiting mass transport due to the presence of a three phase system that is inherently immiscible in each other with liquid/liquid/solid $(\mathrm{L} / \mathrm{L} / \mathrm{S})$ interfaces. With the intention of ameliorating conditions for a reaction system containing $\mathrm{L} / \mathrm{L}$ immiscible reactants, the ability of select metal alkoxides to act as a phase transforming advanced catalytic materials were studied. In this regard, the ability of transition-metal alkoxides to initially act as a homogeneous catalyst and then phase-separate into a heterogeneous form was elucidated. Specifically, the study targeted ascertaining the effects of different metal groups of alkoxide catalysts on the performance (yield and selectivity) toward transesterification. Eight different metals were tested. Transesterification was done using identical weights of metal alkoxides, i.e., $1 \%$ of the total weight of the reactants. Studies confirm that active sites of metal alkoxides can catalyze the transesterification reaction. It was observed that, a maximum ester yields were observed with titanium isopropoxide and yttrium isopropoxide catalysts. The selectivity of all transition metal alkoxide catalysts toward component fatty acid methyl esters was the same.
\end{abstract}

Keywords: Transition Metal Alkoxides, Transesterification, Catalysts, Transport

\section{Introduction}

Reactions involving immiscible reactants are challenging to perform due to limitations associated with mass transport. Nevertheless, there are numerous instances where two immiscible liquid reactants (i.e., polar and non-polar liquids) are needed to be brought in contact with a catalyst -- such as in esterification[1,2] transesterification[3-6], etherification [7,8], and hydrolysis[9-11]. Reaction progression in such systems is critically affected by mass transfer issues associated with unavailability of sufficient interfacial area. The issues are exacerbated when a catalyst is required.

The L/L incompatibility could be ameliorated by introducing a liquid surfactant[12]; and if this surfactant is a catalyst, such a catalytic surfactant is expected to significantly help transport aspects of such immiscible systems. Nevertheless, homogeneous catalysts complicate down-stream product separation. Heterogeneous catalyst on the other hand, though easy to separate subsequent to completion of the reaction, introduces transport limitations which results in meager reaction performance.
Accordingly, the primary goal of this study was to evaluate the catalytic performance of select transition metal alkoxides that can initially act as homogenous catalysts during transesterification and then affords the ability to separate the spent catalyst as a heterogeneous solid from the reaction medium once the reaction is complete (as a result of concurrent condensation-polymerization).

Transesterification is an acid or alkaline catalyzed reaction[13]. Due to superior activity and favorable economics, the most commonly used industrial catalysts are alkaline (sodium and potassium) hydroxides/alkoxides and inorganic acids (primarily sulfuric acid). An advantage of these catalysts is that these are homogeneous with the product medium which favors transport aspects. Nevertheless, the most significant drawback of these homogeneous catalysts is complication of the downstream product separation and purification steps[14].

To circumvent downstream separation issues, several heterogeneous catalysts have been suggested. These include: metal oxides such as $\mathrm{ZrO} 2-\mathrm{SiO} 2, \mathrm{KOH} / \mathrm{ZrO} 2-\mathrm{SiO} 2$, Co2O3-SiO2, Mo2O5-SiO2, Na2O-SiO2[15, 16]; metals such as nickel powder, palladium powder, cast iron shavings, 
steel shavings [16, 17]; zeolites [18-20]; ion exchange resins $[15,21,22]$; and salts such as sulfated zirconia [1, 23, 24]. However, it has been widely reported that heterogeneous catalysts require more intensive reaction conditions [25-27] and longer reaction times [1, 4, 27] as compared to the homogeneous counterparts. By contrast, homogeneous basic catalysts give conversions near $100 \%$ at temperatures at or below $650 \mathrm{C}$ in less than 3 hours [28]. The meager performance of heterogeneous catalysts can be primarily attributed to substrate transport issues.

Studies were conducted to develop a catalytic process that can harness the advantages of aforementioned homogeneous catalysts while addressing the $\mathrm{L} / \mathrm{L}$ compatibility issue and additionally that can alleviate the separation issue. The hypothesis was that metal alkoxide catalysts, in their monomeric (or simple oligomeric forms) can act as transesterification catalysts as well as amphiphiles (due to the presence of hydrophobic hydrocarbon tail-groups and oxygen-bound metal groups that are hydrophilic in metal alkoxides). A previous study, established the effectiveness of titanium isopropoxide based monomers, oligomers and polymers as transesterification catalysts [29]. A subsequent study looked at how hydrocarbon tail group complexity affects the ester yields [30]. This study focuses on understanding: 1) The catalytic effect of the metal group of different transition-metal alkoxides toward transesterification; 2) their selectivity toward component fatty acid esters formation; 3) if differences (in terms of yields) are observed, the underlying reasons; and 4) verification of the ability of theses alkoxides to polymerize and separate from the medium subsequent to the reaction.

\section{Materials and Methods}

\subsection{Materials}

The transesterification reaction was carried out in a high pressure thermal reactor (4570-Parr instrument, Moline, IL USA.) with maximum operating temperature rating of $500 \mathrm{oC}$ and pressure of $5000 \mathrm{psi}$. The reactor consisted of a $500 \mathrm{ml}$ vessel fitted with a magnetic drive stirrer with maximum speed of $2000 \mathrm{rpm}$ (tachometer module with accuracy $\pm 10 \mathrm{rpm}$ ). The reactor was used in the batch mode. Degummed soybean oil was purchased in bulk from STE Oil Company, San Marcos, TX USA. The typical fatty acid profile of soybean oil is: C16:0-11\%, C18:0-4\%, C18:1-23\%, C18:2-54\% and C18:3-8\% [31].

Isopropoxides were selected as alkoxide group donors due to the wide availability in different metal forms (as opposed to methoxides or ethoxides). Isopropoxides of nickel, iron, lanthanum, chromium, yttrium, and zirconium were purchased from Chemat Technology Inc. Titanium isopropoxide, sodium isopropoxide and sodium methoxide (control) were purchased from Sigma-Aldrich Corp. Isopropanol and methanol were purchased from EMD chemicals Inc. Pure biodiesel (control) was purchased from SoyGold (Ag environmental products, LLC, Omaha, NE
USA).

\subsection{Catalyst Preparation}

All the catalysts were prepared via ultrasonic mixing with respective alcohols. Isopropoxides were diluted in isopropanol while methoxides were diluted in methanol. The sonication system (UP400S, Hielscher ultrasound technology) is capable of producing $24,000 \mathrm{kHz}$ waves with a power output of $400 \mathrm{~W}$ for this purpose. As different metal alkoxides have different numbers of alkoxide groups, monomers were chosen to ensure homogeneity. Catalysts were prepared according to previously developed methods [30]. Here it should be noted that not all the metal isopropoxides dissolve in isopropanol. In all cases, fine power forms of metal alkoxides were used for the reaction and when not dissolved, the suspension of metal alkoxide in isopropanol was used. Titanium, iron, zirconium, yttrium, sodium isopropoxides dissolved in isopropanol while nickel, chromium, and lanthanum isopropoxides didn't.

\subsection{Transesterification}

The transesterification reactions were carried out in the aforementioned high pressure reactor. In order to initiate the transesterification reaction, $1 \%(\mathrm{w} / \mathrm{w})$ of the catalyst was infused into the reaction chamber containing triglyceride immediately after the contents reached the designated temperature $(200 \mathrm{oC})$ through a high pressure liquid pump (Eldex 5790, Eldex laboratories Inc. Napa, CA). It was noticed that when the reactor reached $200 \mathrm{oC}$, the pressure increased up to $10 \mathrm{psi}$. Alcohol to oil ratio of 3:1 (molar basis) was used for the transesterification reaction. Isopropanol was used as the esterification reagent for all of the reactions with isopropoxide catalysts. Methanol was used as the esterification alcohol when sodium methoxide was used as the catalyst (control). Samples were drawn from the reactor at 30-minute intervals up to 3 hours. The samples drawn were cooled immediately in order to cease the reaction from progressing further. Then, the samples were centrifuged at 10,000 RPM at $12 \mathrm{oC}$ to separate products (alkyl esters and glycerol) and any catalyst. The centrifuge system used (Sorvall Legend 23 R- Thermo Scientific) has maximum rated speed of $24,000 \mathrm{rpm}$ and minimum temperature of (-) $4 \mathrm{oC}$.

The top fraction of the products was analyzed for esters using gas chromatography (GC-6850 Agilent Technologies, Santa Clara, CA USA). Auxiliary analyses for confirmation of the products were carried out via GC Mass spectroscopy (GC-MS 7890 Agilent Technologies, Santa Clara, CA USA). The gas chromatograph was calibrated with the respective alkyl ester standards each time prior to obtaining quantitative yields. Alkyl ester standards (pertinent to methyl, isopropyl, esters of C 16:0, 18:0, 18:1, 18:2, 18:3 fatty acid alkyl esters) were purchased from $\mathrm{Nu}-\mathrm{Chek}$ Prep Inc.(Elysian MN 56028, USA).

The GC method utilized (for isopropyl esters) detection is as follows: 
Inlet Temperature

Split ratio

Injection volume

Column flow (Helium)

FID temperature

H2 Flow

Air flow

Makeup gas (Nitrogen)

Oven program 50oC hold $1 \mathrm{~min}$, to $200 \mathrm{oC}$ at 25

$\mathrm{oC} / \mathrm{min}$, hold $3 \mathrm{~min}$, to $230 \mathrm{oC}$ at 3

oC/min, hold $18 \mathrm{~min}$.

Column $30 \mathrm{~m}$ x $0.25 \mathrm{~mm}$ x $0.25 \mu \mathrm{m}$ (DB-Wax)

The fatty acid alkyl esters yield was calculated via Chemstation software (Agilent technologies). The area under the peak from the FID chromatogram corresponded to the concentration of that component. These concentrations were determined using calibrations with pure ester standards along with an internal standard (C-12 ester).

The results were statistically analyzed by Design Expert software.

\subsection{Catalyst Characterization}

The acidity and basicity of the catalyst was determined by the Hammett indicator method [32]. The Hammett indicator method is a fast and widely accepted method for determining the basicity and acidity of solid catalysts. However, it should be noted that this method is only accurate for qualitative analysis due to the potential issues associated with indicator molecules diffusing in to the micro-pores when present [32, 33].

About 1-2 $\mathrm{ml}$ of sample containing the catalyst was shaken with 5 drops of Hammett indicator in isopropanol solution and left to equilibrate for $3 \mathrm{~h}$. In the Hammett indicator method, the base strength is quoted as being stronger than the weakest indicator which exhibits a color change, but weaker than the strongest indicator that produces no color change $[32,33]$. In these experiments, the following Hammett indicators were used: Neutral red (NR) (pKa 6.8), Methyl red (MR) (pKa 4.8), P-dimethylaminoazobenzene (DM) (pKa 3.3), Crystal violet $(\mathrm{CV})$ (pKa 0.8), Phenolphthalein (PH) (pKBH+ $8.2)$, Nile blue (NB) (pKBH+ 10.1), Tropaeolin (TR) $(\mathrm{pKBH}+11)$ and 2,4-dinitroaniline (DN) (pKBH+ 15). All the indicators were purchased from Sigma-Aldrich Co. LLC.

\subsection{Catalyst Selectivity}

There are many definitions for calculating selectivity in literature. In the context of this work, the following formula was used [34]:

$$
\text { Product Selectivity }=\frac{\text { Moles of a productcomponent }}{\text { Total moles of product }} \times 100 \%
$$

The results were statistically analyzed by Design Expert software.

\section{Results and Discussion}

Experiments were carried out in two stages. During the first stage, the ester yields were evaluated using select transition-metal alkoxides as catalysts. Then, the ester yields were normalized according to the number of alkoxide ligands bound to the metal. By doing this, it is possible to eliminate the yield differences due to a variable number of alkoxide active sites. This also would help compare results with prior work that used metal alkoxides $[35,36]$. It should be noted that the effect of thermal reactions (not-catalytic reactions due to high temperature) was studied previously and it was observed that no significant transesterification occurred during a time span of 3 hours at 200oC [30].

Figure 1 depicts the ester yields of various metal isopropoxides with time. It was observed that titanium and yttrium isopropoxide showed almost similar ester yields after 3 hours. However, titanium demonstrated a faster reaction rate at the beginning (while the rate diminished toward to end of the reaction period). Yttrium, on the other hand, had the same rate throughout the reaction period.

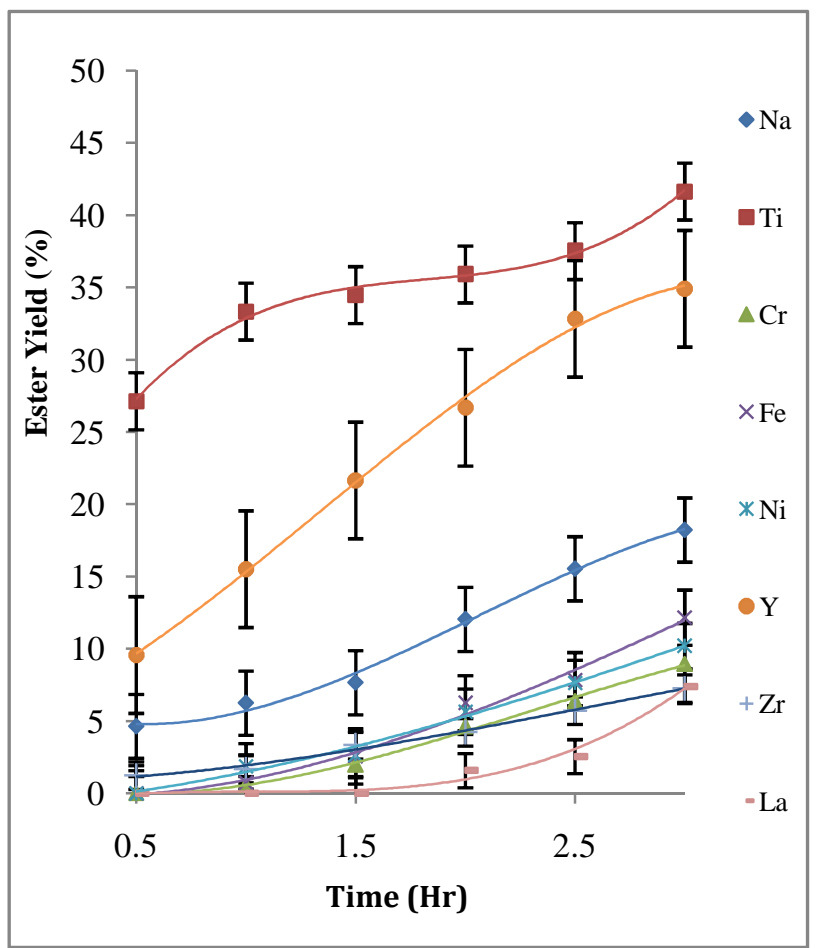

Figure 1. Ester yields with respect to time for alkoxide catalysts with different metal groups.

$\mathrm{Na}$ isopropoxide was used as the control to compare the performance of other isopropoxides since alkaline metal alkoxides are the most widely used catalyst for transesterification.[15, 37] It is interesting to observe that $\mathrm{Ti}$ $(42 \%)$ and $\mathrm{Y}$ isopropoxides $(35 \%)$ yielded higher amounts of esters as compared to $\mathrm{Na}(18 \%)$. ANOVA table for ester yields is depicted in figure 2. The statistical analysis shows that the ester yields of metal isopropoxides are significantly different after 3 hours of reaction time. The analysis depicts that the ester yields for $\mathrm{Cr}, \mathrm{Fe}, \mathrm{La}, \mathrm{Zr}, \mathrm{Ni}$ isopropoxides are not significantly different after three hrs. Also none of the above isopropoxides displayed comparable ester yields to Ti 
or Y.

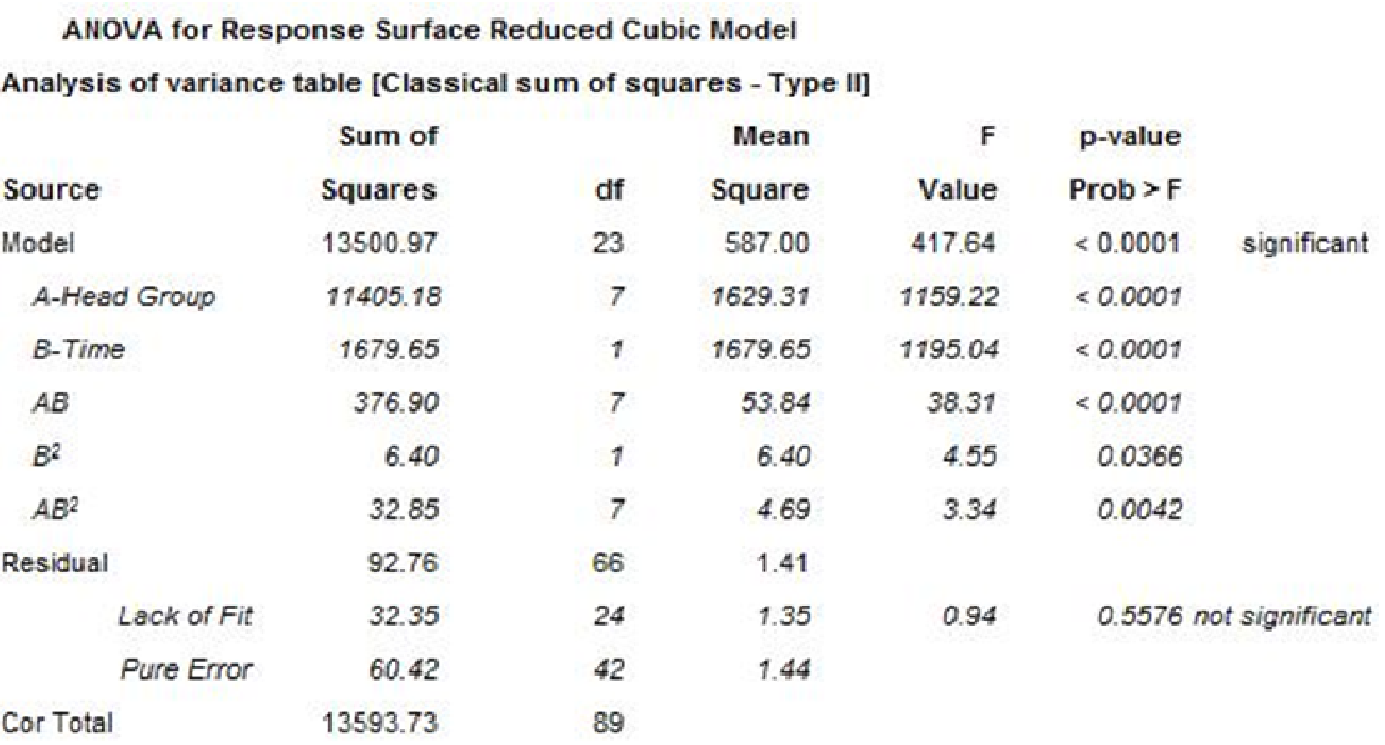

Figure 2. ANOVA table for yield analysis with different metal alkoxides.

The ester yields depicted in figure 1 are based on identical weights of catalyst. It should be noted that the catalyzing reaction can take place at the Ti centre (where Ti acting as a Lewis acid) or at the alkoxide centre (where alkoxide acting as a Brønsted base, depending on the metal used) [30]. Presuming that the basic reaction is more dominant, the yield variability may be as a result of the varying number of alkoxide ligands and not due to the nuances of metal centre. To correct this situation we have normalized the yields to "per moles of ligand groups attached". For example titanium isopropoxide has 4 alkoxide ligands attached to the titanium centre while nickel has only two. In order to rectify this discrepancy, the ester yields per alkoxide branch were calculated (figure 3). For this comparison, we have based the yields of all alkoxides with respect to the one that gave the highest ester yield (assigning it a 100\% activity) per mole of active branches in the reaction.

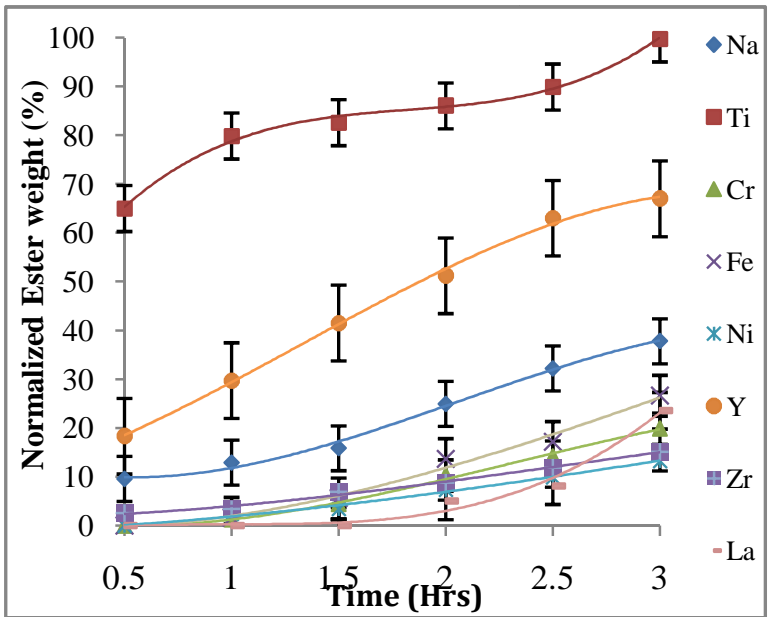

Figure 3. Normalized ester yields (\% weight) with respect to the number of active ligands.
It is evident that even after normalizing for the number of active sites, titanium based catalyst renders the highest ester yield per mole of active branch. Nevertheless, only titanium and yttrium resulted in significant ester yields. It is important to note that the performance of sodium isopropoxide was still much inferior to that of titanium and yttrium counterparts.

It is important to compare the transition-metal alkoxide catalytic activity to alkali metal catalytic activity since alkali metals are the most widely used in industry. Sodium and potassium alkoxides are the most prevalent catalysts for transesterification in biodiesel industry [37]. Accordingly, the transesterification ability of sodium isopropoxide was compared to that of sodium methoxide (Figure 4). It is clear that the catalytic ability of sodium methoxide is far superior to that of the sodium isopropoxide. Sodium methoxide had a maximum ester yield of $83 \%$ just after 30 minutes of reaction. This yield is several-fold higher than that of the isopropoxide counterpart (which yielded less than 20\% esters even after 3 hours of reaction). In alkali-metal alkoxide homologous series, molecular complexity increases with increasing carbon number in alkoxide. Thus, there is higher covalence between the metal atom and the oxygen atom for higher carbon alkoxide groups. This makes it harder for the higher-carbon-numbered alkoxide to participate in branch confirmation reactions. However, it should be noted that alkali-metal methoxides are metastable compounds compared to alkali-metal isopropoxides. Therefore, polymerizing to its oxide form is less favorable in methoxide than isopropoxide [38]. Accordingly, methoxides (to a large extent) would stay in solution as a homogenous catalyst while propoxides will oligomerize and eventually polymerize becoming a solid (heterogeneous catalyst). The propensity of the alkali-metal methoxide to stay in solution (homogeneous with the oil-phase and alcohol-phase) 
coupled with all active sites being available throughout the entirety of the reaction period may have instigated the comparatively higher ester yields in comparison to isopropoxides. Isopropoxides, on the other hand, may have forgone some of the active sites for oligomerization making the number of active site available for transesterification progressively lesser as the reaction progressed - prompting comparatively lesser ester yields than methoxides.

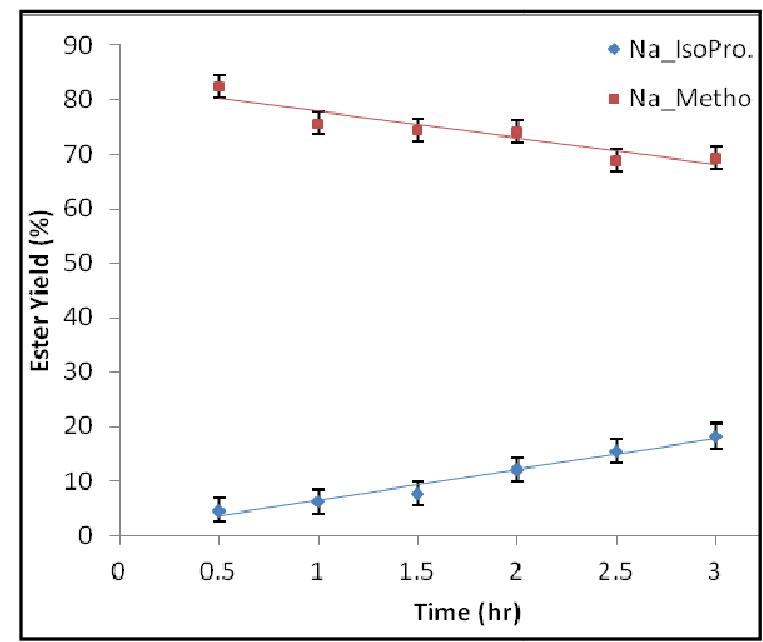

Figure 4. Ester yields of sodium isopropoxide and sodium methoxide.

It is interesting to note that titanium methoxide, resulted in an ester yield of $64 \%$ after 3 hours of reaction. The titanium methoxide data were collected in a previous study [30]. The analysis suggests that the activity of titanium methoxide as a transesterification catalyst is not far from that of the sodium counterpart.

Although sodium methoxide caused a reduction of ester yields with time, the yields were much higher as compared to that of sodium isopropoxide. A possible explanation for low ester yields when using sodium methoxide in this experiment (although sodium methoxide is the most active catalyst for transesterification) is the likely thermal degradation of produced esters. Results suggest that transesterification reaction occurs at a fast rate during the first 30 minutes of reaction time (our first sample was drawn only after $30 \mathrm{~min}$ ). Nevertheless, results from Figure 4 depict that after three hours of reaction, the yields have decreased (when sodium methoxide was used as the catalyst). It is likely that when sodium methoxide was used at $200 \mathrm{oC}$, the yield of transesterification products peaking before $30 \mathrm{~min}$.

The selectivities of select catalysts towards component esters are shown in figure 5. It is evident that all three catalysts, sodium, titanium and yttrium isopropoxides had comparable selectivities toward component fatty acids. At the onset of the reaction, the selectivity for stearate and linolinate was zero for all the catalysts (depicting that the product spectrum had only C16:0, C18:1 and C18:2 fatty acids). Ti was more selective for C18:1fattyacids, compared to $\mathrm{Na}$ and $\mathrm{Y}$. Sodium, on the other hand, had a higher selectivity towards C18:2 linoleate ester $(63 \%)$ at the onset of the reaction.

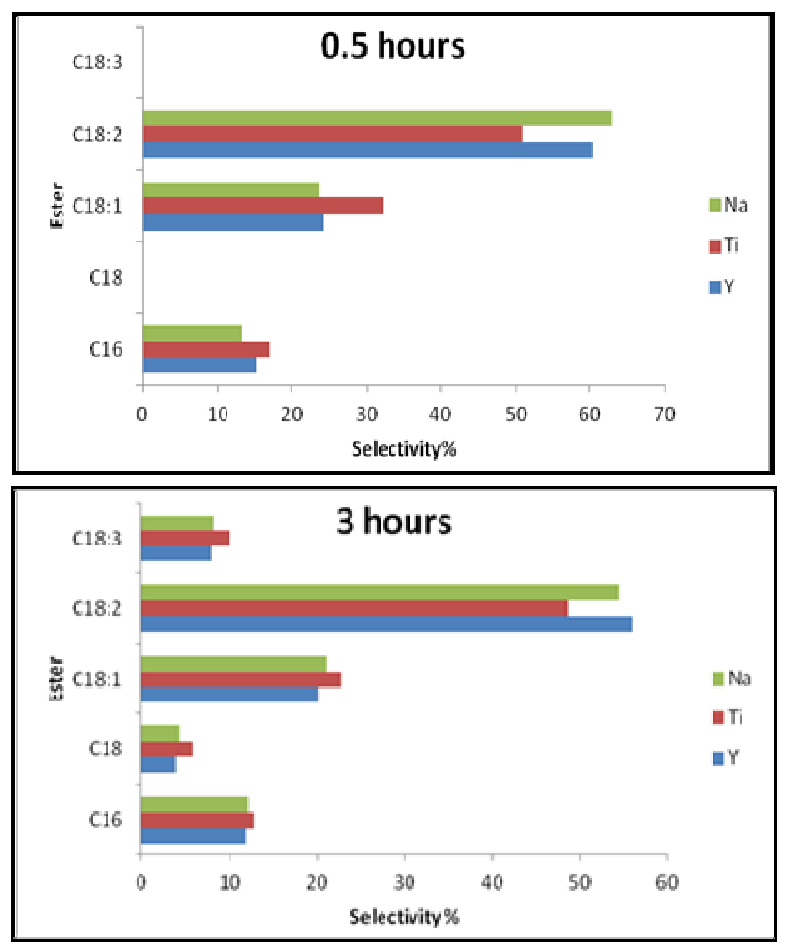

Figure 5. Selectivity of different metal groups for component esters with respect to time.

At the end of three hours, stearate and linolinate esters also appeared in the product mix. At this point, titanium had a higher selectivity toward all the major fatty acids except C18:2 linoleate ester. It was noted that ester composition of triglycerides closely followed the selectivity numbers. Literature suggest that, fatty acids C16-12\%, C18- 5\%, C18:1- 25\%, C18:2- 52\%, C18:3- $6 \%$ by weight in soybean oil. The overall conclusion subsequent to the statistical analysis is that after three hours of reaction time, all the three catalysts are equally selective for component fatty acid hydrolysis.

An interesting observation was that although all metal isopropoxides at the onset of the reaction were homogeneous with the reaction medium, subsequent to the reaction, most transition-metal isopropoxides were separable as a heterogeneous product. Figure 6 depicts that alkali metal catalyst cannot be separated (or visible) after vigorous centrifugation of the content. Nevertheless, most transition-metal catalysts were able to be separated from esters and glycerol. Transition-metal alkoxides can polymerize via alcohol condensation at higher temperatures [10,39-42]. The observations allude that the transition-metal alkoxides went through a concurrent polymerization reaction while participating in the catalysis of the transesterification reaction. The fortuitous outcome is a fully separable heterogeneous catalyst from the reaction medium at the end of the reaction. These observations are in line with findings from our previous studies that partially polymerized transition-metal alkoxides act as transesterification catalysts $[29,30]$.As a practical significance, it should be noted here 
that the heterogeneous forms of all the isopropoxides tested can be relatively easily transformed chemically to its original monomeric form for reuse [38].

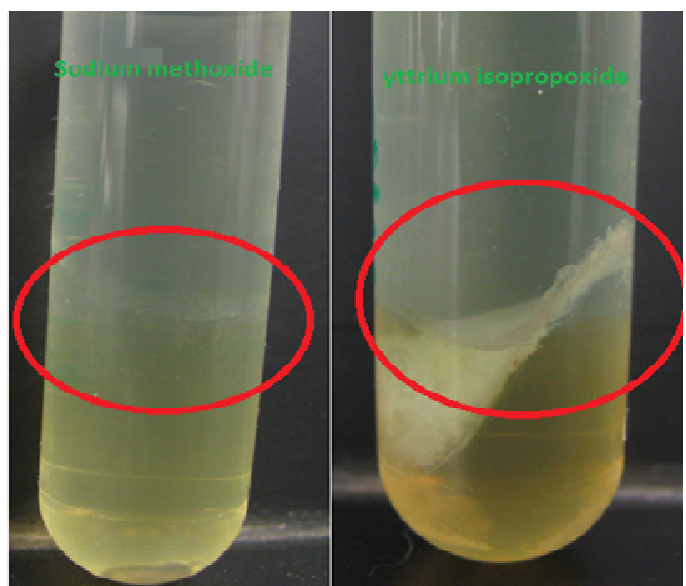

Figure 6. Transesterification products after centrifugation - homogeneous alkali metal alkoxide catalyst (left) and heterogeneous transition-metal alkoxide catalyst (right).

The studies allude that the early transition-metals are significantly more catalytically active towards transesterification than the others tested. It is evident that metal alkoxides in group III and IV in d-bock rendered the highest transesterification yields. The reasons for this could be several-fold. At the beginning of the reaction, it is likely that all metal alkoxides would be in their monomeric (or the thermodynamically most stable - simplest forms). The group IVB metals possess the highest number of alkoxide ligands coordinated around the metal center. This translates to a higher number of catalytic active sites per mole participating the reaction and thus higher ester yields when compared to other metals in the same period.

However, as the transesterification reaction progresses, it is evident that the alkoxides oligomerize either via alcohol condensation or water condensation routes. It should be noted that all the metal alkoxides oligomerize even with traces of water present - and when they do oligomerize forming metal-oxo-alkoxides, the ligands arrange according to least complex formation. The main principle is that metal atoms attempt to achieve the highest coordination with the lowest possible molecular complexity [43]. Accordingly, when comparing the transition-metal alkoxide homologs along the transition-metal series, oligomerized Ti-oxo-alkoxides would have the highest number of alkoxide ligands attached per metal center. This explains the significantly higher activity of early transition-metals (Ti and Y) as compared to late transition metals. Interestingly, zirconium, though an early transition-metal did not perform as well as Ti or Y most likely due to the reaction progressing via an acid-catalyzed route (as opposed to $\mathrm{Ti}$ and $\mathrm{Y}$ progressing via a basic route) as identified by the Hammett indicator studies described below.

\subsection{Catalyst Characterization}

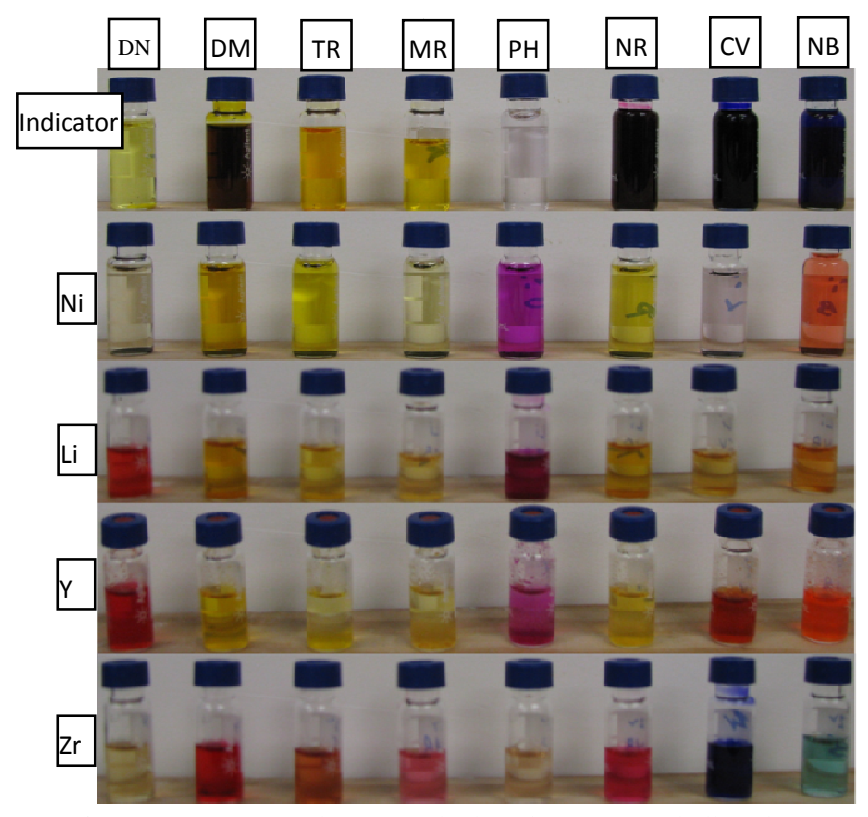

Figure 7. Hammett indicator method on different metal alkoxides.

Catalyst matrix after subjecting to Hammett indicator staining is depicted in Figure 7. Based on the color changes, it was observed that not all the metal alkoxides tested were basic. For example zirconium isopropoxide displayed strongly acidic properties. Interestingly, zirconium rendered very low ester yields throughout the experiment. Titanium and yttrium isopropoxides, on the other hand, were highly basic and gave high of ester yields. Table1 presents the conjugate-base basicity of different metal alkoxides used in this experiment. It is clear from the analysis that Lithium isopropoxide which is an alkali metal alkoxide is strongly basic. It is evident that catalysts that showed strong basicity favored transesterification as opposed to the acidic counterparts.

Table 1. Acidity/Basicity of selected metal alkoxide catalysts.

\begin{tabular}{lll}
\hline Catalyst & $\begin{array}{l}\text { pKa value } \\
\text { Method) }\end{array}$ \\
\hline Lithium isopropoxide & 11.2 \\
Zirconium isopropoxide & 3.25 \\
Nickel isopropoxide & 11.2 & \\
Titanium isopropoxide & 10.1 \\
Iron isopropoxide & 6.8 \\
Yttrium isopropoxide & 11.2 \\
\hline
\end{tabular}

This analysis indicates that transition-metal isopropoxides can catalyze transesterification using either alkoxide ligands located at the exterior of the metal complex via base catalyze route or using the (acidic) metal center depending on the acidity/basicity of the complex.

\section{Conclusions}

This study looked at the catalytic behavior of transition-metal alkoxides (initially introduced into the reactant mixture in monomeric form while phase changing into polymeric forms) toward transesterification. Maximum 
ester yields observed were $42 \%$ for titanium isopropoxide, $35 \%$ for yttrium isopropoxide (both after 3 hours of reaction time) and $18 \%$ for sodium isopropoxide with $1 \%$ catalyst by weight. In comparison, the ester yield was $83 \%$ with sodium methoxide (alkaline earth alkoxide) after only 30 minute of reaction time. The study confirmed that of the transition-metal isopropoxides tested, titanium and yttrium had the highest activity toward transesterification. The study also confirmed the superior catalytic ability of sodium alkoxides. It was established that the catalytic ability of the methoxide was far superior to that of the isopropoxides. After correcting for the number of active sites present per mole of catalyst, it was evident that the activity of titanium and yttrium isopropoxides was far superior to that of the sodium counterpart - and was an unexpected. The selectivity of select metal isopropoxides ( $\mathrm{Na}, \mathrm{Ti}$ and $\mathrm{Y}$ ) revealed that all catalysts had equal selectivity toward component fatty acid esters formation.

An interesting finding is that transition-metal alkoxides undergo a concurrent polymerization reaction while catalyzing the transesterification reaction. Although all the catalysts were homogeneous with the reaction medium at the onset of the reaction, as a result of this simultaneous polymerization, the transition-metal alkoxide catalysts polymerized/solidified and were easily separable from the reaction medium subsequent to completion of the reaction.

Further analysis suggests that transition-metal isopropoxides can catalyze transesterification using either alkoxide ligands located at the exterior of the metal complex via base catalyzed route or using the (acidic) metal center depending on the acidity/basicity of the complex. Ni, Ti and Yi isopropoxides displayed basic properties and likely progressed the transesterification reaction via the base catalyzed route whereas $\mathrm{Zr}$ and $\mathrm{Fe}$ isopropoxides progressed the reaction via the acidic pathway. This study confirms that ability of transition metal alkoxides to be used as a phase-transforming catalytic material.

\section{Acknowledgements}

This material is based upon work supported by the National Science Foundation under Grant No. CBET 0924900 .

\section{References}

[1] Kim, H., et al., Development of Heterogeneous Catalyst System for Esterification of Free Fatty Acid Contained in Used Vegetable Oil. Studies in Surface Science and Catalysis, 2004. 153: p. 4.

[2] Sejidov, F.T., Y. Mansoori, and N. Goodarzi, Esterification reaction using solid heterogeneous acid catalysts under solvent-less condition. Journal of Molecular Catalysis A: Chemical, 2005. 240(1-2): p. 186-190.

[3] Puna, J.F., et al., Advances on the development of novel heterogeneous catalysts for transesterification of triglycerides in biodiesel. Fuel, 2010. 89(11): p. 3602-3606.
[4] Gomes, J., et al., Development of heterogeneous catalysts for transesterification of triglycerides. Reaction Kinetics and Catalysis Letters, 2008. 95(2): p. 273-279.

[5] Benjapornkulaphong, S., C. Ngamcharussrivichai, and K. Bunyakiat, Al2O3-supported alkali and alkali earth metal oxides for transesterification of palm kernel oil and coconut oil. Chemical Engineering Journal, 2009. 145(3): p. 468-474.

[6] Chen, H., et al., Biodiesel production by the transesterification of cottonseed oil by solid acid catalysts. Frontiers of Chemical Engineering in China, 2007. 1(1): p. $11-15$.

[7] Yadav, G.D. and M.S. Krishnan, Etherification of $\hat{\mathrm{I}}^{2}$-Naphthol with Alkanols Using Modified Clays and Sulfated Zirconia. Industrial \& Engineering Chemistry Research, 1998. 37(8): p. 3358-3365.

[8] Kim, J.W., et al., Preparation and characterization of zeolite catalysts for etherification reaction. Catalysis Today, 2003. 87(1-4): p. 195-203.

[9] Kröcher, O. and M. Elsener, Hydrolysis and oxidation of gaseous HCN over heterogeneous catalysts. Applied Catalysis B: Environmental, 2009. 92(1-2): p. 75-89.

[10] Iloukhani, H., S. Azizian, and N. Samadani, Hydrolysis of Sucrose by Heterogeneous Catalysts. Reaction Kinetics and Catalysis Letters, 2001. 72(2): p. 239-244.

[11] Lykourinou-Tibbs, V., A. Ercan, and L.-J. Ming, Iron(III)-Chelex resin complex as a prototypical heterogeneous catalyst for phosphodiester hydrolysis. Catalysis Communications, 2003. 4(10): p. 549-553.

[12] Nandi, A., A. Mehra, and D.V. Khakhar, Coalescence in a Surfactant-less Emulsion under Simple Shear Flow. Fluid Mechanics and Transport Phenomena, 2005. 52(3): p. 10.

[13] Wenzel, B., et al., Modelling Chemical Kinetics of Soybean Oil Transesterification Process for Biodiesel Production: An Analysis of Molar Ratio between Alcohol and Soybean Oil Temperature Changes on the Process Conversion Rate. Bioautomation, 2006. 5: p. 10.

[14] Zhou, W. and D.G.B. Boocok, Phase Distributions of Alcohol, Glycerol, and Catalyst in the Transesterification of Soybean Oil. JAOCS, 2006. 83(12): p. 6.

[15] Pinto, A.C., et al., Biodiesel: An overview. J. Braz. Chem. Soc., 2005. 16 (6B): p. 18.

[16] Suppes, G., et al., Transesterification of Soybean Oil with Zeolite and Metal Catalysts. Applied Catalysis A: General, 2004. 257: p. 11.

[17] Dasari, M., M. Goff, and G. Suppes, Noncatalytic Alcoholysis Kinetics of Soybean Oil. Journal of the American Oil Chemists' Society, 2003. 80(2).

[18] Serio, M., et al., Transesterification of Soybean Oil to Biodiesel by Using Heterogeneous Basic Catalysts. Ind. Eng. Chem. Res., 2006. 45: p. 6.

[19] Leclercq, E., A. Finiels, and C. Moreau, Transesterification of Rapeseed Oil in the Presence of Basic Zeolites and Related Solid Catalysts. JAOCS, 2001. 78(11): p. 5.

[20] Cantrell, D.G., et al., Structure-reactivity correlations in $\mathrm{MgAl}$ hydrotalcite catalysts for biodiesel synthesis. Applied Catalysis A: General, 2005. 287: p. 8. 
[21] Shibasaki-Kitakawa, N., et al., Biodiesel Production Using Anionic Ion-Exchange Resin as Heterogeneous Catalyst. Bioresource Technology, 2006. In press.

[22] Reis, S.C.M.d., et al., Transesterification of Brazilian Vegetable Oils with Methanol over Ion-Exchange Resins. JAOCS, 2005. 82(9): p. 5.

[23] Furuta, S., H. Matsuhashi, and K. Arata, Biodiesel Fuel Production with Solid Superacid Catalysis in Fixed Bed Reactor under Atmospheric Pressure. Catalysis Communications, 2004. 5(12): p. 3.

[24] Chen, H., et al., Biodiesel production by the transesterification of cottonseed oil by solid acid catalysts. Front. Chem. Eng. China, 2007. 1(1): p. 5.

[25] Yan, S., S.O. Salley, and K.Y. Simon Ng, Simultaneous transesterification and esterification of unrefined or waste oils over $\mathrm{ZnO}-\mathrm{La} 2 \mathrm{O} 3$ catalysts. Applied Catalysis A: General, 2009. 353(2): p. 203-212.

[26] Noiroj, K., et al., A comparative study of KOH/Al2O3 and $\mathrm{KOH} / \mathrm{NaY}$ catalysts for biodiesel production via transesterification from palm oil. Renewable Energy, 2009. 34: p. 1145-1150.

[27] Di Serio, M., et al., Transesterification of Soybean Oil to Biodiesel by Using Heterogeneous Basic Catalysts. Industrial \& Engineering Chemistry Research, 2006. 45(9): p. 3009-3014.

[28] Vicente, G., M. Martinez, and J. Aracil, Integrated biodiesel production: a comparison of different homogeneous catalysts systems. Bioresource Technology, 2004. 92: p. 9.

[29] Nawaratna, G., S.D. Fernando, and S. Adhikari, Response of Titanium-Isopropoxide-Based Heterogeneous Amphiphilic Polymer Catalysts for Transesterification. Energy \& Fuels, 2010. 24(8): p. 4123-4129.

[30] Nawaratna, G., R. Lacey, and S.D. Fernando, Effect of hydrocarbon tail-groups of transition metal alkoxide based amphiphilic catalysts on transesterification. Catalysis Science \& Technology, 2012.

[31] Eren, T., et al., Hydroxymethylation and polymerization of plant oil triglycerides. Journal of Applied Polymer Science, 2004. 91(6): p. 4037-4046.

[32] Hammett, L.P. and M.A. Paul, A Series of Simple Basic Indicators. III. The Zero Point of the Acidity Function Scale.
Journal of the American Chemical Society, 1934. 56(4): p. 827-829.

[33] Xie, W. and X. Huang, Synthesis of Biodiesel from Soybean Oil using Heterogeneous KF/ZnO Catalyst. Catalysis Letters, 2006. 107(1): p. 53-59.

[34] Ferdous, D., A.K. Dalai, and J. Adjaye, Comparison of product selectivity during hydroprocessing of bitumen derived gas oil in the presence of $\mathrm{NiMo} / \mathrm{Al} 2 \mathrm{O} 3$ catalyst containing boron and phosphorus. Fuel, 2006. 85(9): p. 1286-1297.

[35] Curran, M.D., T.E. Gedris, and A.E. Stiegman, Catalysis of Silicon Alkoxide Transesterification by Early Transition Metal Complexes. Chemistry of Materials, 1998. 10(6): p. 1604-1612.

[36] Kawakami, K., et al., Doubly Activated Supramolecular Reaction: Transesterification of Acyclic Oligoether Esters with Metal Alkoxides. The Journal of Organic Chemistry, 2010. 76(3): p. 875-881.

[37] Gerpen, J.V., B. Shanks, and R. Pruszko, Biodiesel Production Technology, 2004.

[38] N.Y. Turova, et al., The Chemistry of Metal Alkoxides2002, New Yourk: Kluwer Academic Publishers.

[39] Ritala, M., et al., Titanium isopropoxide as a precursor in atomic layer epitaxy of titanium dioxide thin films. Chemistry of Materials, 1993. 5(8): p. 1174-1181.

[40] Bradley, D.C. and J.M. Thomas, Metal Alkoxides as Precursors for Thin-Film Growth [and Discussion]. Philosophical Transactions of the Royal Society of London. Series A, Mathematical and Physical Sciences, 1990. 330(1610): p. 167-171.

[41] Bradley, D.C., Metal alkoxides as precursors for electronic and ceramic materials. Chemical Reviews, 1989. 89(6): p. $1317-1322$.

[42] Orgel, L.E., An Introduction to transition-metal chemistry ligand-field theory. 1 ed1960, New York: John Wiley \& Sons Inc.

[43] Bradley, D.C. Chemistry and reactions of metal alkoxides. 1992. Wiley. 